\title{
DAS MINDESTALTER DER STADT NYSA NACH DER LITERARISCHEN ÜBERLIEFERUNG
}

\section{Gerd SACHS *}

\author{
Schlüsselwörter: Antiochos $\bullet$ Hellenismus $\bullet$ Karien $\bullet$ Nysa $\bullet$ Strabon $\bullet$ Stephanos von Byzantion
}

Zusammenfassung: Der Aufsatz befasst sich mit den auf uns überkommenen antiken Schriftquellen zu der Stadt Nysa am Mäander, um hieraus in Ergänzung zu dem archäologischen Befund Hinweise auf das Alter der Stadt zu finden. Hauptquellen sind der Geograf und Historiker Strabon sowie der Grammatiker Stephanos von Byzantion. Strabon nennt mehrere berühmte Männer, die in der Philosophenschule der Stadt gelehrt haben. Aus ihren Lebensdaten kann man entnehmen, dass Nysa bereits in der zweiten Hälfte des 2. Jh. v. Chr. bestanden hat. Stephanos berichtet, welche Städte von Antiochos in Karien gegründet worden sind, und nennt hierunter auch Nysa. Ob sich sein Bericht auf Antiochos I. oder Antiochos III. bezieht, kann allerdings nicht mit Sicherheit festgestellt werden. Immerhin ergibt sich hieraus, dass die Stadt entweder in der ersten Hälfte oder im letzten Viertel des 3. Jh. v. Chr. gegründet worden sein muss.

\section{EDEBİ KAYNAKLAR IŞIĞINDA NYSA KENTİNİN ERKEN TARİHİ}

Keywords: Antiokhos $\bullet$ Hellenizm $\bullet$ Karia $\bullet$ Nysa $\bullet$ Strabon $\bullet$ Byzantionlu Stephanos

Özet: Bu makale Nysa antik kenti ile ilgili antik edebi kaynakları ve arkeolojik belgeleri inceleyerek Nysa antik kentinin geçmişini incelemektedir. Bu bağlamda en önemli kaynağımız coğrafyacı ve tarihçi Strabon ile Byzantionlu Stephanos'dur. Strabon Nysa'da eğitim görmüş birçok ünlü kişiden bahseder. Söz konusu bu kişilerin yaşadığı tarihlerden Nysa antik kentinin M.Ö. 2. yüzyllın 2. yarısından itibaren var olduğu anlaşılmaktadır. Stephanos, Antiokhos tarafindan Karia'da kurulmuş olan kentleri ve bu kentler arasında da Nysa'yı saymaktadır. Stephonos'un burada I. Antiokhos'u mu yoksa III. Antiokhos'u mu kastettiği açıkça belli değildir. Buna karşılık Nysa antik kenti M.Ö. 3. yüzyllın ilkyarısında ya da son çeyreğinde kurulmuş olmalıdır. 
Der stoische Historiker und Geograph Strabon von Amaseia (geb. 64/63 v. Chr., gest. nach 23 n. Chr.) verbrachte eine Zeit seiner Jugend in Nysa, wo er an einer Philosophenschule der Stoa studierte ${ }^{1}$. In Buch XIV. 1 Kap. 43 seiner Geographie beschreibt er die Stadt wie folgt:

„Nysa liegt an der Messogis, sich größtenteils an den Berg anlehnend. Sie ist ganz. wie eine Doppelstadt; denn ein eine Schlucht bildendes Flussbett trennt sie, über das von der einen Seite eine die beiden Stadtteile verbindende Brücke führt, während die andere durch ein Amphitheater geschmückt ist, unter dem sich ein verborgener Durchfluss für das aus der Schlucht kommende Wasser befindet. Neben dem Theater gibt es awei Anböhen; unterbalb der einen liegt das Gymnasion der Jugend und unterbalb der anderen die Agora und das Gerontikon; im Süden unterbalb der Stadt erstreckt sich die Ebene, ebenso wie bei Tralleis. ${ }^{2}$ "

Nach dieser Beschreibung war Nysa in den frühen 40er Jahren des 1. Jh. v. Chr. eine römische Provinzstadt mit den üblichen öffentlichen Einrichtungen.

In Kap. 46 schildert Strabon die Gründungslegende: Hiernach seien die Brüder Athymbros, Athymbrados und Hydrelos aus Lakedaimon gekommen und hätten nach ihnen benannte Städte gegründet; später seien diese aber menschenleer geworden; aus ihrer Vereinigung sei Nysa entstanden. Die Nysaier würden nach wie vor den Athymbros für den Gründer ihrer Stadt halten.

\footnotetext{
1 Zur allgemeinen Literatur über die Stadt s. von Diest 1913, Magie 1950, 989ff.; Bean, 1971, 221 ff.; Kaletsch 2000, 1075f.; İdil 1999; Kadıŏglu 2006, 3ff.

2 Eigene Übersetzung.
}

In Kap. 48 schreibt er über berühmte Männer aus Nysa Folgendes:

„Berühmte Männer von Nysa waren der stoische Philosoph Apollonios, der beste unter den Scbülern des Panaitios, und Menekrates, der Schüler des Aristarchos; dann Aristodemos, der Sohn von jenem, den ich, noch sebr jung, als hochbejahrten Greis zu Nysa börte. Sostratos ferner, des Aristodemos Bruder, und ein anderer Aristodemos, der Vetter des Sostratos, welcher den Pompeius Magnus unterrichtete, waren vorzügliche Grammatiker; der meinige aber lebrte auch die Redekunst und bielt sowobl zu Rhodos als in seiner Vaterstadt zwei Schulen, des Morgens die Redekunst, des Abends die Sprachwissenschaft lebrend; zu Rom jedoch, wo er die Söhne des Pompeius unterrichtete, beschränkte er sich auf eine Schule der Sprachwissenschaft. ${ }^{3}$ "

Panaitios, der Begründer der mittleren Stoa, lebte von ca. 185 bis 109 v. Chr. In den Jahren 129 - 109 v. Chr. leitete er die Philosophenschule. Bei dem von Strabon genannten Schüler kann es sich nur um den Stoiker Apollonios von Tyros handeln, der ein mehrbändiges Werk über das Leben Zenons verfasst hat ${ }^{4}$. Der bedeutende alexandrinische Grammatiker Aristarchos von Samothrake lebte von ca. 216 - 144 v. Chr. ${ }^{5}$ Sein Schüler Menekrates dürfte hiernach in der zweiten Hälfte des 2. Jh. v. Chr. in Nysa gelehrt haben, was auch dadurch bestätigt wird, dass dessen Sohn Aristodemos in den frühen 40er Jahren des 1. Jh. v. Chr. ,als hochbejahrter Greis“ den jungen Strabon unterrichtet hat.

\footnotetext{
Übersetzung A. Forbiger.

Diog. Laert., 7, 1, 2, 6, 24.

Montanari 1996, $1090 \mathrm{ff}$.
} 
Durch die Nennung von Apollonios und Menekrates als berühmten Männern von Nysa ist literarisch belegt, dass Nysa nicht erst zur Zeit Strabons, sondern bereits in der zweiten Hälfte des 2. Jh. v. Chr. eine Stadt mit einer Philosophenschule war. Ab dem Jahre 129 v. Chr. gehörte Nysa zu der römischen Provinz Asia Minor, zuvor war das Gebiet Bestandteil des Reiches von Pergamon. Aus dem Bericht Strabons ergibt sich also, dass Nysa zumindest seit späthellenistischer Zeit eine bedeutende Stadt gewesen ist.

Nach den derzeitigen Erkenntnissen wird die Stadt Nysa in der antiken Literatur nur noch im 1. Jh. n. Chr. von Plinius Secundus, im 2. Jh. von Ptolemaios, im 3. Jh. von Julius Africanus ${ }^{6}$ und im 6. Jh. von Stephanos von Byzantion erwähnt.

Plinius nennt in Buch $\mathrm{V}$ seiner naturalis historia bei der geographischen Beschreibung Kariens neben zahlreichen anderen Städten Nysa zusammen mit Tralleis ohne weitere Hinweise. versalgelehrte Ptolemaios aus Alexandria verzeichnet in seinem in der zweiten Hälfte des 2. Jh. erschienenen geographischen Katalog vor der Stadt Aphrodisias ebenfalls nur den Namen der Stadt (Nyssa) und ihre nach Längen- und Breitenposition berechnete Lage ${ }^{8}$.

Während sich aus den Erwähnungen bei Plinius und Ptolemaios keine weiteren Erkenntnisse über Nysa entnehmen lassen, können die Städtebeschreibungen des griechischen Grammatikers Stephanos Hinweise auf das Alter der Stadt ergeben. Er

\footnotetext{
6 Grenfell - Hunt 1903, 36-41 Nr. 412 Taf. 5 (col. II 58-68).

7 Plin. nat., 5, 108.

8 Ptol. 5, 2. 18.
}

schreibt in seinem alphabetisch angelegten geographischen Lexikon über Nysa an vier Stellen Folgendes:

s. v. Athymbra:

„Stadt in Karien am Maiandros, eine Gründung des Atbymbros, welche später Nyssa genannt wurde."

s. v. Antiocheia:

„Zehn Städte sind aufgezeichnet, es sind aber mehr. ... Die elfte in Karien, die auch Pythopolis genannt wurde. Dem Antiochos, Sobn des Seleukos erschienen im Traum drei Franen, für die er jeweils eine Stadt in Karien baute; er bielt sie für seine Mutter, seine Frau und seine Schwester und gründete tatsäcblich [drei Städte], die er nach der Schwester Laodike Laodikeia, nach der Ehefrau Nyse Nysa und nach der Mutter Antiochis Antiocheia benannte ".

s. v. Nysai:

„Viele Städte, ... die dritte in Karien."

s. v. Pythopolis:

„Stadt in Karien, welche später Nysa genannt wurde."

Die erste Nennung deckt sich teilweise mit dem Gründungsmythos Strabons und ist mangels weiterer Anhaltspunkte keine sichere historische Aussage. Der zweite Bericht verweist entweder auf Antiochos I. (324 - 261 v. Chr.) oder Antiochos III. (ca. 240 - 187 v. Chr.), wie sich aus dem Vaternamen Seleukou ergibt. Die beiden weiteren Stellen nennen nur den Namen der Stadt, ohne sie historisch einzuordnen.

Welcher Antiochos Nysa gegründet haben soll, lässt sich den Ausführungen des Stephanos nicht mit hinreichender Sicherheit entnehmen; denn sie sind bezüglich der

9 Eigene Übersetzung. 
genannten Personen voller Widersprüche. Antiochos I. war verheiratet mit Stratonike, der früheren Ehefrau seines Vaters Seleukos I., zu der er in Liebe entbrannt war und die er bei seiner Ernennung zum Mitregenten zur Gemahlin erhielt. Dies ist von Plutarch ausführlich überliefert ${ }^{10}$. Seine Mutter hieß Apama, eine Schwester namens Laodike ist nicht bekannt. Sein Urenkel Antiochos III. war in erster Ehe mit Laodike, einer Tochter Mithradates' III. von Pontos verheiratet und hatte von ihr eine Tochter Nysa ${ }^{11}$. Sollte ein Antiochos tatsächlich die Stadt Nysa gegründet haben, muss Stephanos ca. 800 Jahre später die Namen gründlich durcheinander gebracht haben.

Antiochos I. könnte nach dem mit Antigonos Gonatas im Jahre 278 v. Chr. getroffenen Grenzabkommen, als er in Kleinasien ungestört regieren konnte, Nysa gegründet oder den in der Gründungslegende Strabons beschriebenen Synoikismos (Kap. 46) veranlasst haben. Ein zusätzliches Argument für den Wahrheitsgehalt des Berichts von Stephanos zur Gründung Nysas besteht darin, dass die beiden weiteren Orte in Karien, die von ihm ebenfalls als Gründungen des Antiochos genannt werden, östlich von Nysa lokalisiert worden sind. Ein Antiocheia liegt am Dandalas, einem südlichen Nebenfluss des Mäanders etwa 5-6 $\mathrm{km}$ südöstlich von Nazilli. Hier fanden wir im Sommer 2008 zufällig ein Keramikfragment aus der ersten Hälfte des 3. Jh. v. Chr. Die antike Stadt Laodikeia liegt $65 \mathrm{~km}$ weiter östlich unmittelbar nördlich der heutigen Stadt Denizli.

\footnotetext{
10 Plut. Demetrios $38 \mathrm{f}$.

11 Vgl. Bradford-Welles 1962, 439 und 454-55.
}

Aber auch Antiochos III., dessen Regierungszeit im Jahre 223 v. Chr. begann, kommt hiernach als Erbauer der Stadt in Frage; denn mit ihm waren die von Stephanos erwähnten Damen Laodike und Nyse verbunden. Die Keramikfragmente weisen allerdings auf ein früheres Gründungsdatum hin. Sie sind indes zu spärlich, um einen sicheren Schluss ziehen zu können.

Die derzeit bekannten Inschriften sind keine weiteren Quellen für das Alter der Stadt. Ein inschriftlich erhaltener Brief der Könige Seleukos und Antiochos I. aus dem Jahre 281 v. Chr. befasst sich nur mit Privilegien für das westlich der Stadt gelegene Pluto - Heiligtum ${ }^{12}$, das nach Strabons Beschreibung bei dem Dorf Acharaka (etwa $4 \mathrm{~km}$ westlich von Nysa) $\operatorname{lag}^{13}$. Der Brief ist nicht an die Bürger von Nysa gerichtet; der Name der Stadt wird nicht einmal genannt. Der Brief beantwortet indes eine Anfrage der „Athymbrianoi“, also wohl der Nachkommen des Athymbros, der als Stadtgründer in der Gründungslegende von Strabon und auch von Stephanos von Byzantion genannt wird und dessen Name hiermit mittelbar auch inschriftlich bestätigt ist. Diese Inschrift kann mangels weiterer Anhaltspunkte jedoch nicht als Beleg dafür gelten, dass die Stadt Nysa an dem in Übereinstimmung mit der Beschreibung Strabons archäologisch gesicherten Platz bereits zu jener Zeit bestanden hat.

Die zeitlich früheste Inschrift, die sich auf die Stadt Nysa bezieht, stammt aus dem Jahre 88 v. Chr. und beinhaltet einen

\footnotetext{
12 SEG 35, 1094; Brodersen et. al. 1996, 310.

13 Strab. XIV. 1. 44.
} 
Treuebeschluss der Nysäer für Rom im Mithradatischen $\mathrm{Krieg}^{14}$.

Die bisher gesicherten Münzen, die eindeutig Nysa zuzuordnen sind, stammen überwiegend aus römischer Zeit. Die frühesten bekannten Prägungen können zwischen 129 und 111 v. Chr. datiert werden, nachdem Nysa als Stadt der römischen Provinz Asia Minor offensichtlich das Münzrecht erhalten hatte ${ }^{15}$.

Als Ergebnis kann festgehalten werden: Nach der literarischen Überlieferung spricht eine hohe Wahrscheinlichkeit dafür, dass die Stadt entweder von Antiochos I. in der ersten Hälfte des 3. Jh. v. Chr. oder von Antiochos III. im letzten Viertel des 3. Jh. v. Chr. gegründet worden ist. Auf jeden Fall war Nysa spätestens ab der zweiten Hälfte des 2. Jh. v. Chr. eine bekannte Stadt mit einer bedeutenden Philosophenschule.

14 Syll. 741 ; Brodersen et.al. 1999, 505.

15 Regling 1913, 70-103. 


\section{BIBLIOGRAPHIE}

Bean 1971

G.E. Bean, Kleinasien 3. Jenseits des Mäander. Karien mit dem Vilayet Muğla (1971).

Bradford-Welles 1962 C. Bradford-Welles, „Genealogien Pontos und Seleukiden“, in: G. Mann A. Heuß (Hrsg.), Propyläen Weltgeschichte, Bd. 3, 1962, 439, 454-55.

Brodersen et.al. 1996 K. Brodersen - W. Günther - H. Schmitt, Historische griechische Inschriften in Übersetzung II (1996).

Brodersen et.al. 1999 K. Brodersen - W. Günther - H. Schmitt, Historische griechische Inschriften in Übersetzung III (1996).

Grenfell - Hunt 1903

B. P. Grenfell - A. S. Hunt, The Oxyrbynchus Papyri III (1903).

Hiesel - Strocka 2006

G. Hiesel - V.M. Strocka,"Die Bibliothek von Nysa am Mäander: Vorläufiger Bericht über die Kampagnen 2002-2006”, AA 2. Halbband 2006, 90 ff.

İdil 1999

V. İdil, Nysa ve Akharaka. Nysa and Acharaca (1999).

Kadıoğlu 2006

M. Kadıoğlu, Die Scaenae frons des Theaters von Nysa am Maeander. Forschungen in Nysa am Maeander I (2006)

Klaetsch 2000

H. Kaletsch, DNP 8, 2000, 1075f. Nr. 3. (s. v. Nysa).

Magie 1950

D. Magie, Roman Rule in Asia Minor (1950).

Montanari 1996

F. Montanari, “Aristarchos”, DNP 1, 1996, 1090-1094.

Regling 1913

K. Regling, "Überblick über die Münzen von Nysa ad Maeandrum”, in: Nysa ad Maeandrum. Nach Forschungen und Aufnabmen in den Jabren 1907 und 1909, JdI, Ergh.10 (1913) 70-103.

von Diest 1913

W. von Diest, Nysa ad Maeandrum. Nach Forschungen und Aufnahmen in den Jahren 1907 und 1909, JdI, Ergh.10 (1913). 